\title{
In-hospital Mortality and the Predictive Ability of the Modified Early Warning Score in Ghana: Single-Center, Retrospective Study
}

Enoch Joseph Abbey ${ }^{1}$, BSc, MD, MPH; Jennifer S R Mammen ${ }^{1}$, BSc, MD, MPhil, PhD; Samara E Soghoian ${ }^{2}$, BSc, MD, MPH; Maureen A F Cadorette ${ }^{3}$, BSN, MPH, PhD; Promise Ariyo ${ }^{4}$, BSc, MD, MPH

\footnotetext{
${ }^{1}$ Division of Endocrinology, Diabetes and Metabolism, Department of Medicine, Johns Hopkins School of Medicine, Baltimore, MD, United States

${ }^{2}$ Department of Emergency Medicine, New York University Grossman School of Medicine, New York University Langone Health, New York, NY, United States

${ }^{3}$ Occupational and Environmental Health, Department of Environmental Health and Engineering, Johns Hopkins School of Public Health, Baltimore, MD, United States

${ }^{4}$ Division of Adult Critical Care, Department of Anesthesiology and Critical Care Medicine, Johns Hopkins School of Medicine, Baltimore, MD, United States
}

\section{Corresponding Author:}

Enoch Joseph Abbey, BSc, MD, MPH

Division of Endocrinology, Diabetes and Metabolism

Department of Medicine

Johns Hopkins School of Medicine

5501 Hopkins Bayview Circle

Baltimore, MD, 21224

United States

Phone: 19293457001

Email: eabbey1@jhu.edu

\section{Related Articles:}

Preprint (medRxiv): https://www.medrxiv.org/content/10.1101/2020.08.05.20169219v1

Preprint (JMIR Preprints): https://preprints.jmir.org/preprint/24645

Peer-Review Report by Lincoln Sheets (Reviewer AK): https://med.jmirx.org/2021/3/e30787/

Peer-Review Report by John Mogaka (Reviewer BO): https://med.jmirx.org/2021/3/e30785/

Peer-Review Report by Ana Marusic (Reviewer CM): https://med.jmirx.org/2021/3/e30763/

Authors' Response to Peer-Review Reports: https://med.jmirx.org/2021/3/e30790/

\section{Abstract}

Background: The modified early warning score (MEWS) is an objective measure of illness severity that promotes early recognition of clinical deterioration in critically ill patients. Its primary use is to facilitate faster intervention or increase the level of care. Despite its adoption in some African countries, MEWS is not standard of care in Ghana. In order to facilitate the use of such a tool, we assessed whether MEWS, or a combination of the more limited data that are routinely collected in current clinical practice, can be used predict to mortality among critically ill inpatients at the Korle-Bu Teaching Hospital in Accra, Ghana.

Objective: The aim of this study was to identify the predictive ability of MEWS for medical inpatients at risk of mortality and its comparability to a measure combining routinely measured physiologic parameters (limited MEWS [LMEWS]).

Methods: We conducted a retrospective study of medical inpatients, aged $\geq 13$ years and admitted to the Korle-Bu Teaching Hospital from January 2017 to March 2019. Routine vital signs at 48 hours post admission were coded to obtain LMEWS values. The level of consciousness was imputed from medical records and combined with LMEWS to obtain the full MEWS value. A predictive model comparing mortality among patients with a significant MEWS value or LMEWS $\geq 4$ versus a nonsignificant MEWS value or LMEWS <4 was designed using multiple logistic regression and internally validated for predictive accuracy, using the receiver operating characteristic (ROC) curve.

Results: A total of 112 patients were included in the study. The adjusted odds of death comparing patients with a significant MEWS to patients with a nonsignificant MEWS was 6.33 (95\% CI 1.96-20.48). Similarly, the adjusted odds of death comparing patients with a significant versus nonsignificant LMEWS value was 8.22 (95\% CI 2.45-27.56). The ROC curve for each analysis had a C-statistic of 0.83 and 0.84 , respectively. 
Conclusions: LMEWS is a good predictor of mortality and comparable to MEWS. Adoption of LMEWS can be implemented now using currently available data to identify medical inpatients at risk of death in order to improve care.

(JMIRx Med 2021;2(3):e24645) doi: 10.2196/24645

\section{KEYWORDS}

modified early warning score; MEWS; AVPU scale; Korle-Bu Teaching Hospital; KBTH; Ghana; critical care; vital signs; global health

\section{Introduction}

Critical illness is a leading cause of morbidity and mortality in sub-Saharan Africa, including Ghana [1]. Low- and middle-income countries have a disproportionately higher burden of critical illness with over $90 \%$ of global maternal deaths and deaths from trauma and infections [1-3]. In Ghana, the critical care burden is high. Historically, financial investment has been skewed toward primary health care. Less commitment to critical care means that resources for intensive medical care are limited, and their thought-out and appropriate allocation is important [4].

One of the main reasons why patients deteriorate and die in hospitals is delayed recognition of illness severity in understaffed inpatient wards. Early warning tools to help identify patients at the highest risk of death could help countries like Ghana with resource allocation and clinical decision making (Figure 1).

Figure 1. Conceptual framework showing predictors of in-hospital mortality and the role of the modified early warning score (MEWS) among ill patients.

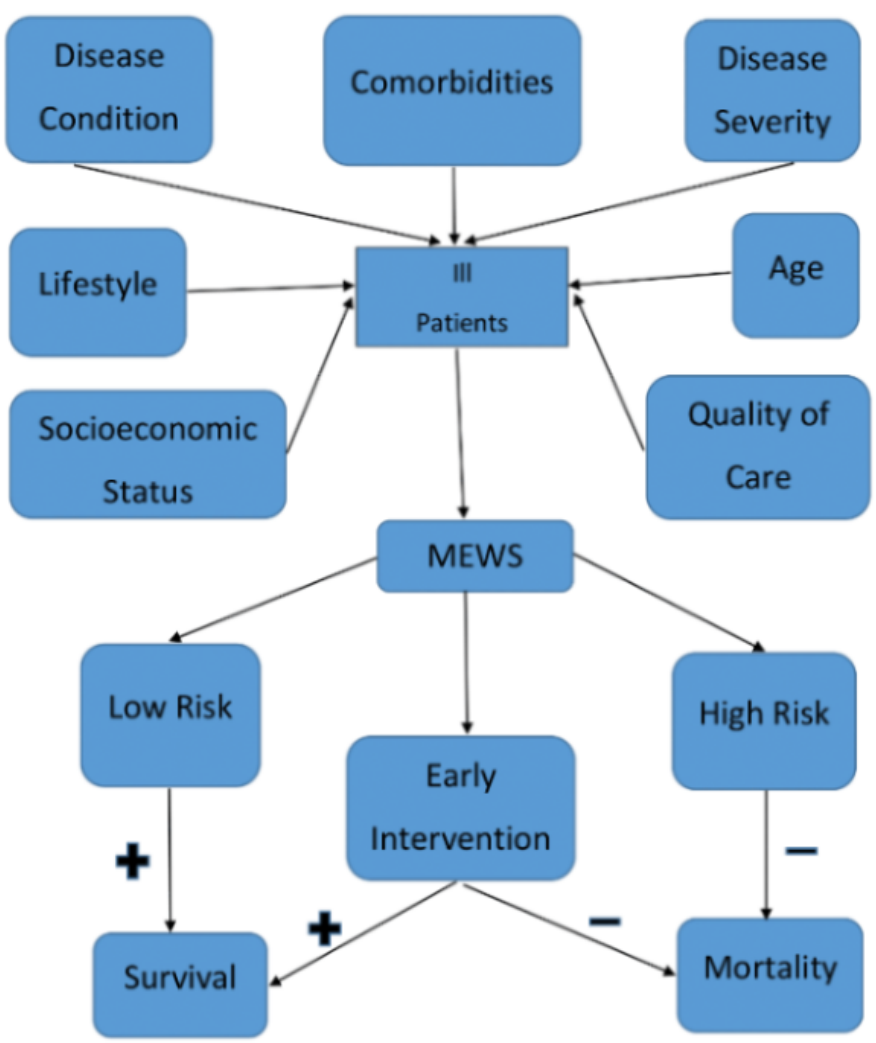

Multiple studies have shown that critical illness and serious adverse events in hospitalized patients are preceded by signs of clinical deterioration in up to $80 \%$ of those affected [5-8]. Therefore, changes in physiological parameters can be used to predict adverse events such as shock, cardiac arrest, death, and unplanned intensive care unit (ICU) admissions [9].

MEWS is a commonly used illness severity score that is calculated by combining five physiologic bedside parameters: systolic blood pressure, heart rate, respiratory rate, temperature, and level of consciousness assessed by the AVPU (alert, [responds to] voice, [responds to] pain, unresponsive) scale or RASS (Richmond Agitation Sedation Scale) score. These four vital signs and the observation of consciousness are individually scored and summed to yield a combined score between 0 and 14, with higher scores representing increased illness severity.

In a systematic review conducted by Smith et al [10] in 2014, early warning scores, including MEWS, had strong predictive ability for death and cardiac arrest within 48 hours in academic urban hospitals in economically advanced countries. Early warning scores have also been shown to provide precise, concise, and unambiguous means of identifying and communicating about clinical deterioration to help clinical staff provide special attention and care to patients who need it most (justifiable appropriation of care) [11]. As a result, scoring 
systems such as MEWS have been adopted in most developed countries and some African countries [12-14].

This study sought to validate the use of MEWS as a clinical decision-making tool to improve early identification of hospitalized medical patients at increased risk for death in Ghana. In addition, since level of consciousness is not routinely recorded in current clinical practice, we aimed to investigate the predictive utility of a limited MEWS (LMEWS) calculation based on vital signs alone. Most studies in similar settings have found that the level of consciousness is generally high (ie, the patient is well oriented) even when other aspects of the MEWS value are abnormal [2]. We therefore hypothesized that the physiologic data currently being monitored in Ghana may be sufficient to improve the early detection of critical illness and help guide resource allocation among inpatients in this setting.

\section{Methods}

\section{Study Design and Population}

This was a retrospective chart review study of hospitalized medical patients, aged $\geq 13$ years, admitted to the Korle-Bu
Teaching Hospital in Accra, Ghana. The Korle-Bu Teaching Hospital is the national hospital of Ghana and the leading tertiary care referral center in the country [15]. Medical inpatients hospitalized there for at least 48 hours whose medical records were still available from the period of January 2017 to March 2019 were included in the study. During this period, the standard practice was to discharge patients in possession of their written medical records; copies were not often retained. This practical limitation accounts for the smaller study size than might be expected for a tertiary facility. Pediatric patients, defined as those aged less than 13 years of age by the Ghana Ministry of Health guidelines, were not included. Patients with more than one hospital admission in the past month, or those who were admitted for conditions other than medical ones, were also excluded (Figure 2). The maximum in-hospital stay was 32 days, and no follow-up data were collected post discharge.

Figure 2. Flow chart demonstrating the creation of the modified early warning score (MEWS) cohort. LMEWS: limited MEWS.

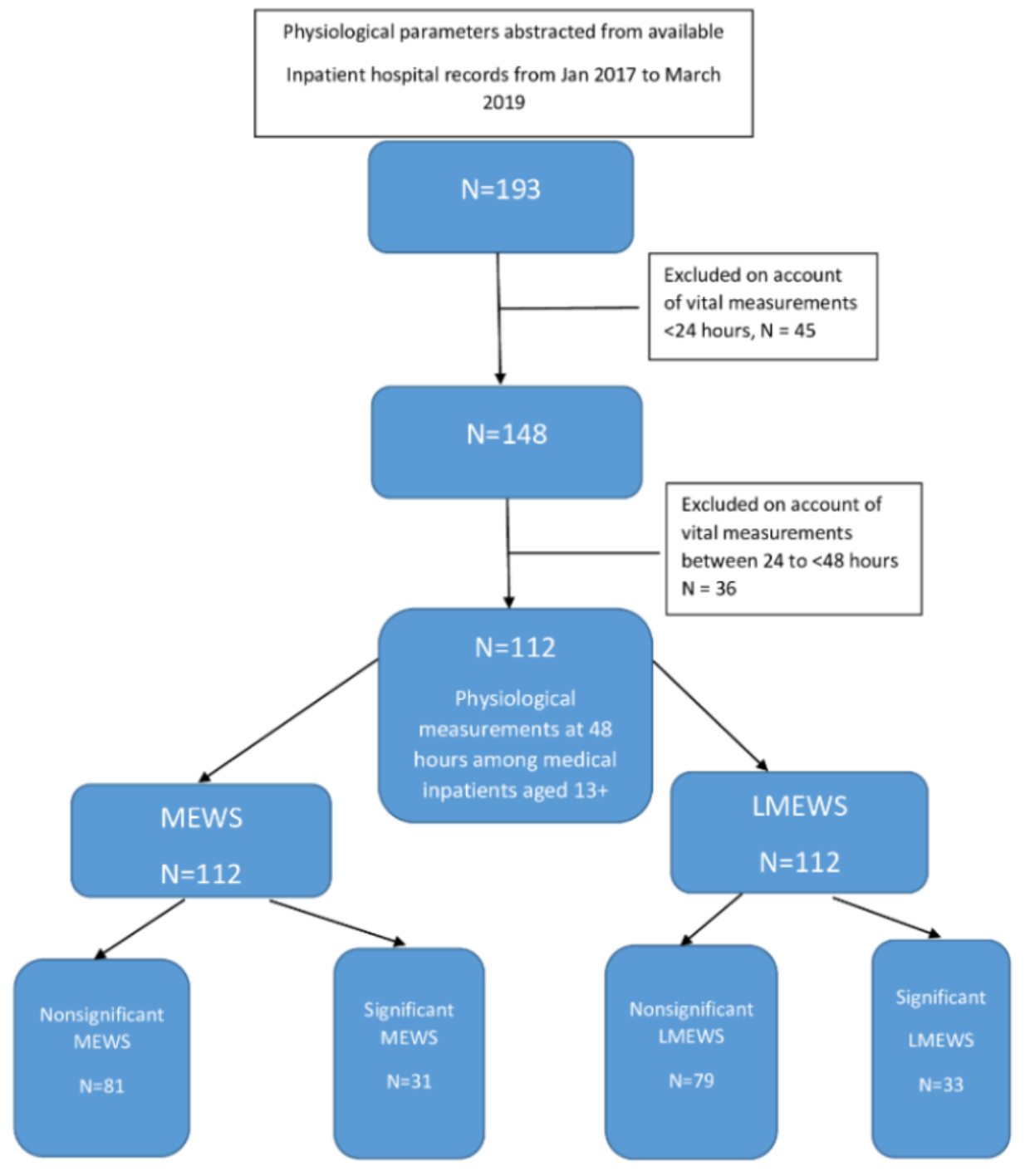


Demographic data were collected to analyze covariates. Patients' vital signs recorded at 48 hours after admission were recoded and scored to generate the LMEWS value, using thresholds as previously described (Table 1) [2]. To compare the utility of LMEWS with the full MEWS in the absence of routine observation of consciousness and recording of AVPU scores, we generated a full MEWS value using imputation by randomly assigning $92 \%$ of the sample to a status of "alert" (AVPU score $=0$ ) and the rest to scores between 1 and 3 . These percentages were determined based on the findings of a study by Subbe et al [2], which used a similar patient population.

Our study was based on the conceptual framework depicted in Figure 1, which identifies correlational patterns of how different events and experiences may predict mortality in a hospitalized patient. A predictive model was designed using multivariable logistic regression and validated for model accuracy to compare patients with significant MEWS to patients with nonsignificant
MEWS, where a significant MEWS was defined as a score $\geq 4$, and a nonsignificant MEWS was defined as a score $<4$ in the absence of the AVPU [3,16,17]. This cut-off did not vary for the LMEWS versus MEWS values since for most individuals the level of consciousness is normal and therefore contributes 0 points to the total MEWS value.

Due to the confidential nature of patient information, and the need to protect anonymity and obtain consent during health record reviews, ethical approval and waiver of documented permission was obtained from the Institutional Review Board (IRB) of Johns Hopkins University, and from the Scientific and Technical Committee (KBTH-STC 00017/2019) and the IRB of the Korle-Bu Teaching Hospital. Although reporting was anonymous, patients' records were not, so researchers involved in data collection and handling also signed a confidentiality clause.

Table 1. Scoring scale for the modified early warning score (MEWS) adopted form Subbe et al [2].

\begin{tabular}{|c|c|c|c|c|c|c|c|}
\hline \multirow[t]{2}{*}{ Physiological parameter } & \multicolumn{7}{|c|}{ MEWS value } \\
\hline & 3 & 2 & 1 & 0 & 1 & 2 & 3 \\
\hline $\begin{array}{l}\text { Systolic blood pressure } \\
(\mathrm{mmHg})\end{array}$ & $<70$ & $71-80$ & $81-100$ & $101-199$ & $-^{\mathrm{a}}$ & $\geq 200$ & - \\
\hline Heart rate (bpm) & - & $41-50$ & $41-50$ & $51-100$ & $101-110$ & $111-129$ & $\geq 130$ \\
\hline Respiratory rate (cpm) & - & - & - & $9-14$ & $15-20$ & $21-29$ & $\geq 30$ \\
\hline Temperature $\left({ }^{\circ} \mathrm{C}\right)$ & - & - & - & $35-38.4$ & - & $\geq 38.5$ & - \\
\hline AVPU $^{\mathrm{b}}$ score & - & - & - & Alert & Reacting to voice & Reacting to pain & Unresponsive \\
\hline
\end{tabular}

${ }^{\mathrm{a}}$ Not applicable.

${ }^{\mathrm{b}}$ AVPU: alert, voice, pain, unresponsive.

\section{Statistical Analysis}

Data were analyzed using STATA (version 15.1, StataCorp LLC). The estimated sample size was determined a priori based on work by Kyriacos et al [18], which yielded a minimum sample size of 46 based on a significance level of .05 , delta value of 0.45 , and power of $80 \%$ to detect clinical deterioration in postoperative patients using MEWS. Post-data collection power analysis was also performed, based on a chi-square test comparing two independent proportions. Based on the resulting analytic sample of 112 participants, with 31 in the significant MEWS category and 81 in the nonsignificant MEWS category, our study achieves a power of $95 \%$ to detect a difference in outcome percentages of at least $37 \%$ between these two groups. Testing for associations with survival to discharge versus in-hospital mortality was conducted using a two-sample $t$ test for each of the individual continuous physiological parameters. The chi-square test was used to test for differences in the proportion of patients with each outcome in the categories of significant versus nonsignificant MEWS and LMEWS. Univariable log-binomial regression analysis was used to estimate unadjusted risk ratios between each predictor and mortality. Multivariable Poisson regression with robust variance was used due to the failure of convergence of the log-binomial regression model. Logistic regression analysis (odds ratio [OR]) was used to identify an appropriate predictive model. A $P$ value of $<.05$ was considered statistically significant. The accuracy of the prediction model was determined using the receiver operating characteristic (ROC) curve and C-statistic (where a C-statistic of 0.5 implies the model performs no better than random chance and a score of 1.00 perfectly discriminates between categories). Adjustment was made for the following potential confounders: age, sex, duration of admission, admission to the ICU, presence or absence of other comorbidities, and the organ system involved in the disease process. The Hosmer-Lemeshow test was used to determine model fit for both the MEWS and LMEWS models, with $P$ values $\geq .05$ implying satisfactory fit. A sensitivity analysis was done using a cut-off of $\geq 5$ to distinguish significant from nonsignificant MEWS and LMEWS values. Missing values were limited to the reason for admission (organ system) and represented $<1 \%(1 / 112)$.

\section{Results}

The sample comprised 112 patients admitted for medical reasons during the study period. Of these, 62\% (69/112) were male with a mean age of 47 years (SD 17.5), and 38\% (43/112) were female with a mean age of 52 years (SD 20) (Table 1). Overall mortality was $41.1 \%(46 / 112)$ and increased with age. Every year increase in age was associated with a $3 \%$ increase in mortality rate after adjusting for MEWS (IRR [incidence rate 
ratio] $=1.03,95 \%$ CI 1.02-1.04). For patients who survived, the most common admission diagnoses were genitourinary system abnormalities $(17 / 65,26.2 \%)$, whereas neurologic conditions were most common among patients who died (18/46, 39\%). The longest length of in-hospital stay was 32 days, with an average of 8 days.

At 48 hours post admission, patients' mean systolic blood pressure was $125 \mathrm{mmHg}$ (SD 2.9), average pulse rate was 91 $\mathrm{mmHg}$ (SD 2), mean axillary temperature was $36.9^{\circ} \mathrm{C}(\mathrm{SD} 0.1)$, and average respiratory rate was $24 \mathrm{cpm}$ (SD 4.7). Only temperature and respiratory rate were individually associated with mortality (Table 2). Physiological parameters measured at 48 hours produced an average LMEWS value of 3 (range 0-11). Imputation of randomly assigned AVPU values increased mean scores by $8 \%$ overall, producing an average MEWS of 3 (range 0-14).

A significant MEWS was associated with a relative risk of 2.01 (95\% CI 1.33-3.04) for death in the univariable analysis, while a significant LMEWS had a relative risk of 2.19 (95\% CI 1.46-3.30) in the univariable analysis (Table 3).

Table 2. Showing baseline characteristics.

\begin{tabular}{|c|c|c|c|}
\hline Characteristic & Survival to discharge $(\mathrm{n}=66)$ & Death in hospital $(n=46)$ & $P$ value $^{\mathrm{a}}$ \\
\hline Sex (male), n (\%) & $45(68.2)$ & $24(52.2)$ & .09 \\
\hline Age (years), n (\%) & & & $<.001$ \\
\hline $25-64$ & $46(69.7)$ & $27(58.7)$ & \\
\hline$\geq 65$ & $7(10.6)$ & $18(39.1)$ & \\
\hline Disease type by system involved, n (\%) & & & .01 \\
\hline Cardiopulmonary & $15(23.1)$ & $13(28.3)$ & \\
\hline Neuroendocrine & $11(16.9)$ & $18(39.1)$ & \\
\hline Hemaoncological & $11(16.9)$ & $1(2.2)$ & \\
\hline \multicolumn{4}{|c|}{ Physiological parameter at 48 hours, mean (SD) } \\
\hline Systolic blood pressure (mmHg) & $127.8(29.4)$ & $120.7(32.1)$ & .23 \\
\hline Pulse rate (bpm) & $89(17.6)$ & $94(18.1)$ & .17 \\
\hline Axillary temperature $\left({ }^{\circ} \mathrm{C}\right)$ & $36.7(0.7)$ & $37.3(1.2)$ & .002 \\
\hline Respiratory rate $(\mathrm{cpm})$ & $23(4.7)$ & $25(6.9)$ & .03 \\
\hline Average length of admission & $7(6.3)$ & $8(7)$ & .60 \\
\hline
\end{tabular}

${ }^{\text {a }} P$ values obtained via the $t$ test and the chi square test.

Table 3. Multivariable logistic regression of death using full modified early warning score (MEWS) and the limited MEWS (LMEWS).

\begin{tabular}{lll}
\hline Covariate & MEWS, odds ratio (95\% CI) & LMEWS, odds ratio (95\% CI) \\
\hline Age & $1.08(1.04-1.12)$ & $1.08(1.04-1.12)$ \\
Sex (male) & $0.44(0.16-1.23)$ & $0.40(0.14-1.13)$ \\
MEWS (significant) & $6.33(1.96-20.49)$ & $8.22(2.45-27.56)$ \\
Duration of admission & $0.99(0.93-1.07)$ & $1.01(0.94-1.08)$ \\
Diseased organ system & $0.59(0.31-1.13)$ & $0.59(0.31-1.12)$ \\
\hline
\end{tabular}

The death rate calculated by the Poisson regression after adjusting for only age was 2.02 (95\% CI 1.40-2.91) times higher in patients with a significant MEWS compared to those with a nonsignificant MEWS. The death rate for a significant MEWS value using LMEWS was 2.13 (95\% CI 1.48-3.07) times that of nonsignificant MEWS after adjusting for age.

In the multivariable predictive model adjusting for age, sex, duration of admission, admission to the ICU, organ system involved, and comorbidities, the odds of death among patients with a significant MEWS was 6.33 (95\% CI 1.96-20.50) times that of patients with a nonsignificant MEWS. The death rate among patients with a significant LMEWS was 8.2 (95\% CI
2.5-27.6) times that of patients with a nonsignificant LMEWS in the multivariable analysis. The best multivariable regression model was selected based on the Akaike Information Criteria, with a value of 116.4. The odds of death for every year increase in age was $8 \%$ (OR 1.08, 95\% CI 1.04-1.12). Other covariates were not statistically significant.

Both MEWS and LMEWS were found to have good discrimination based on the ROC curves, with a C-statistic of 0.833 and 0.838 , respectively (Figures 3 and 4 ), using a cut-off of $\geq 4$. The Hosmer-Lemeshow goodness-of-fit test yielded $P$ values of .16 and .25 for MEWS and LMEWS, respectively, 
implying that our model fits the data well (the null hypothesis being that the prediction model is correctly specified).

Sensitivity analyses using a significant MEWS or LMEWS cut-off score of $\geq 5$ yielded a multivariable OR of 12.4 (95\% CI 2.5-61.2) and 15.1 (95\% CI 2.5-91.8), respectively. The ROC curves for MEWS and LMEWS was found to be 0.838 and 0.840 , respectively, when a cut-off of $\geq 5$ was adopted, as captured in Figures 5 and 6 . The Hosmer-Lemeshow test to assess goodness of fit yielded $P$ values of .51 versus .77 for MEWS and LMEWS, respectively, when a cut-off of $\geq 5$ was used.

Figure 3. Receiver operator characteristic (ROC) curve for the modified early warning score (MEWS) using a cut-off of 4.

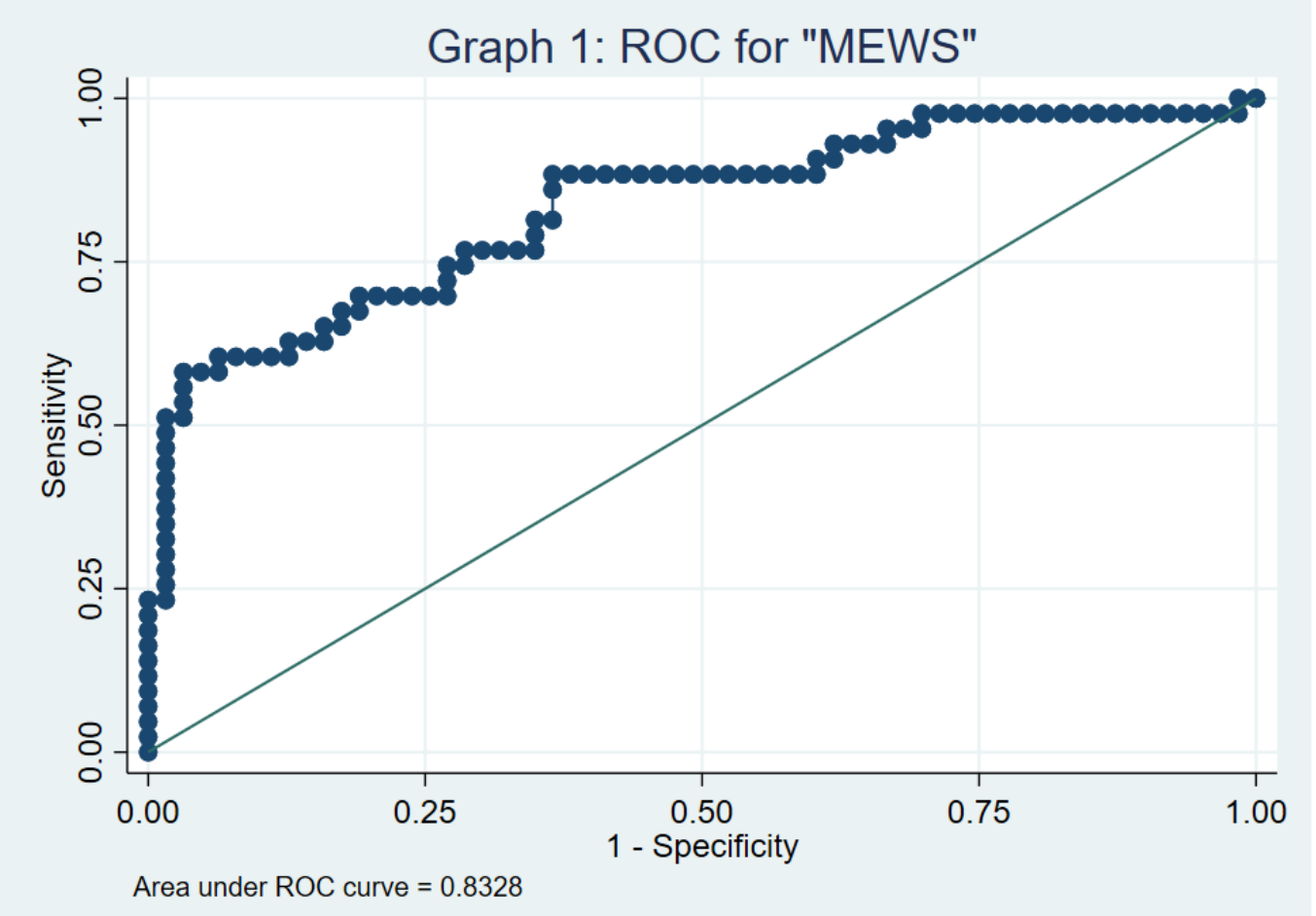

Figure 4. Receiver operator characteristic (ROC) curve for the limited modified early warning score (LMEWS) using a cut-off of 4.

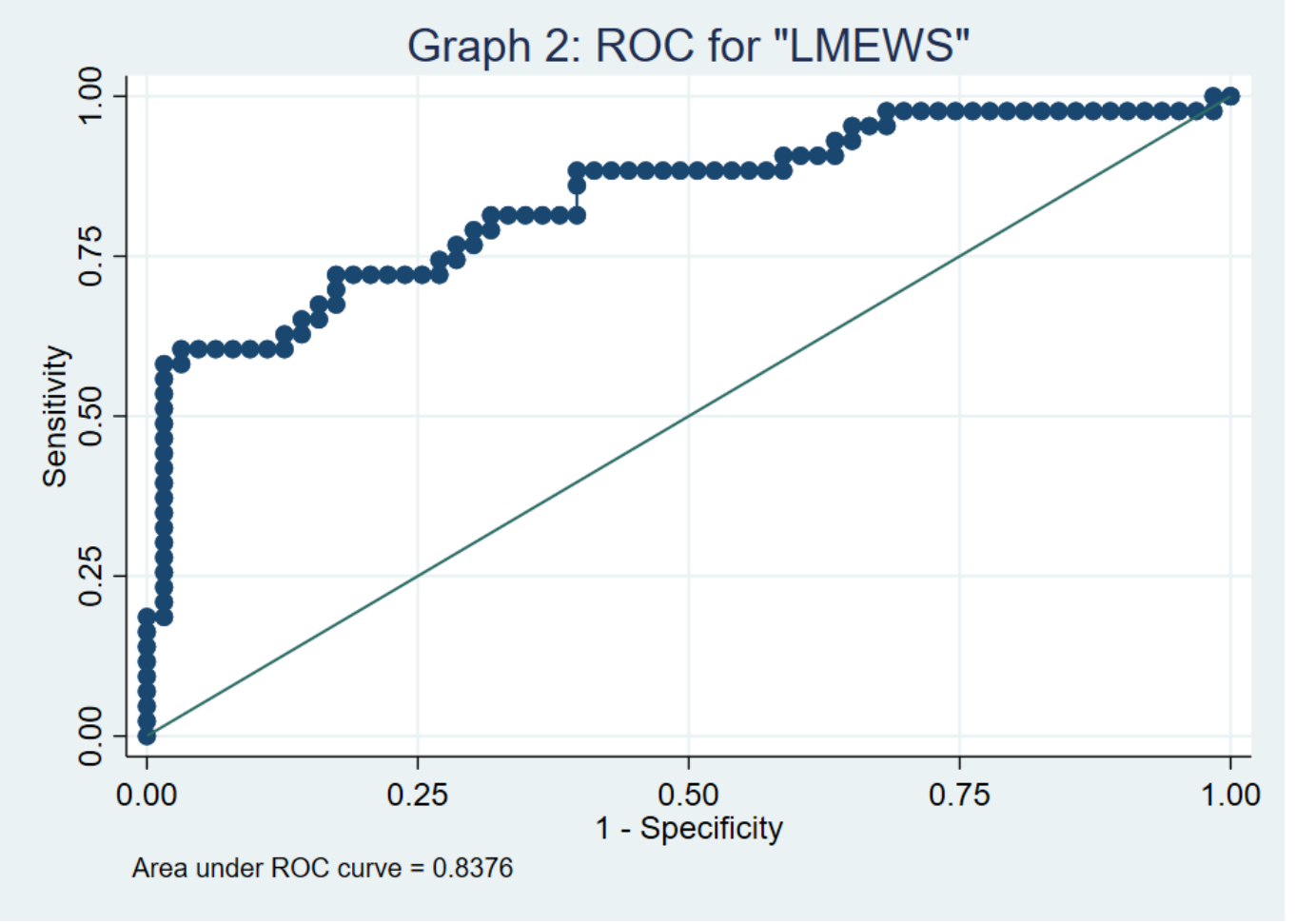


Figure 5. Receiver operator characteristic (ROC) curve for the modified early warning score (MEWS) using a cut-off of 5.

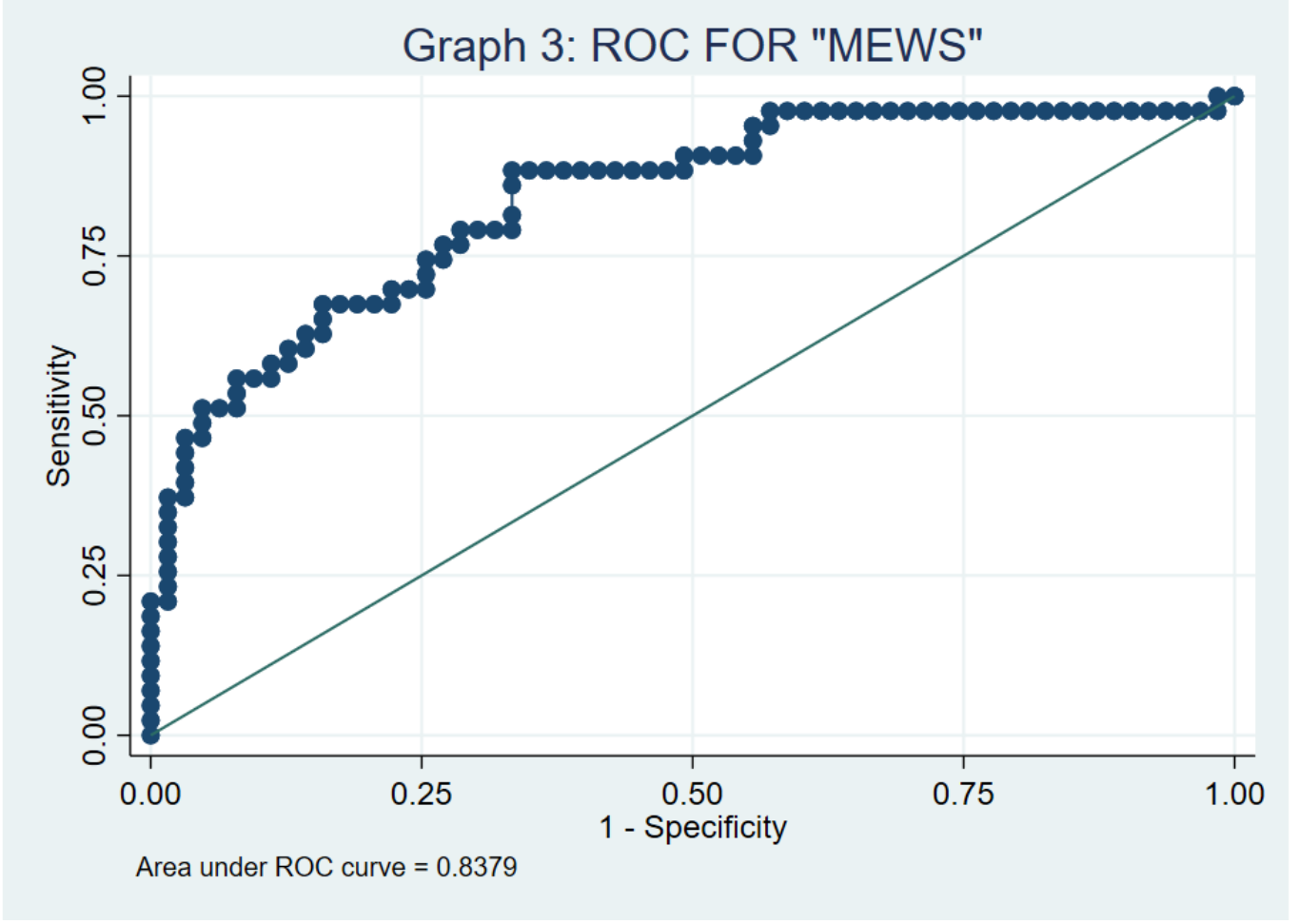

Figure 6. Receiver operator characteristic (ROC) curve for the limited modified early warning score (LMEWS) using a cut-off of 5.

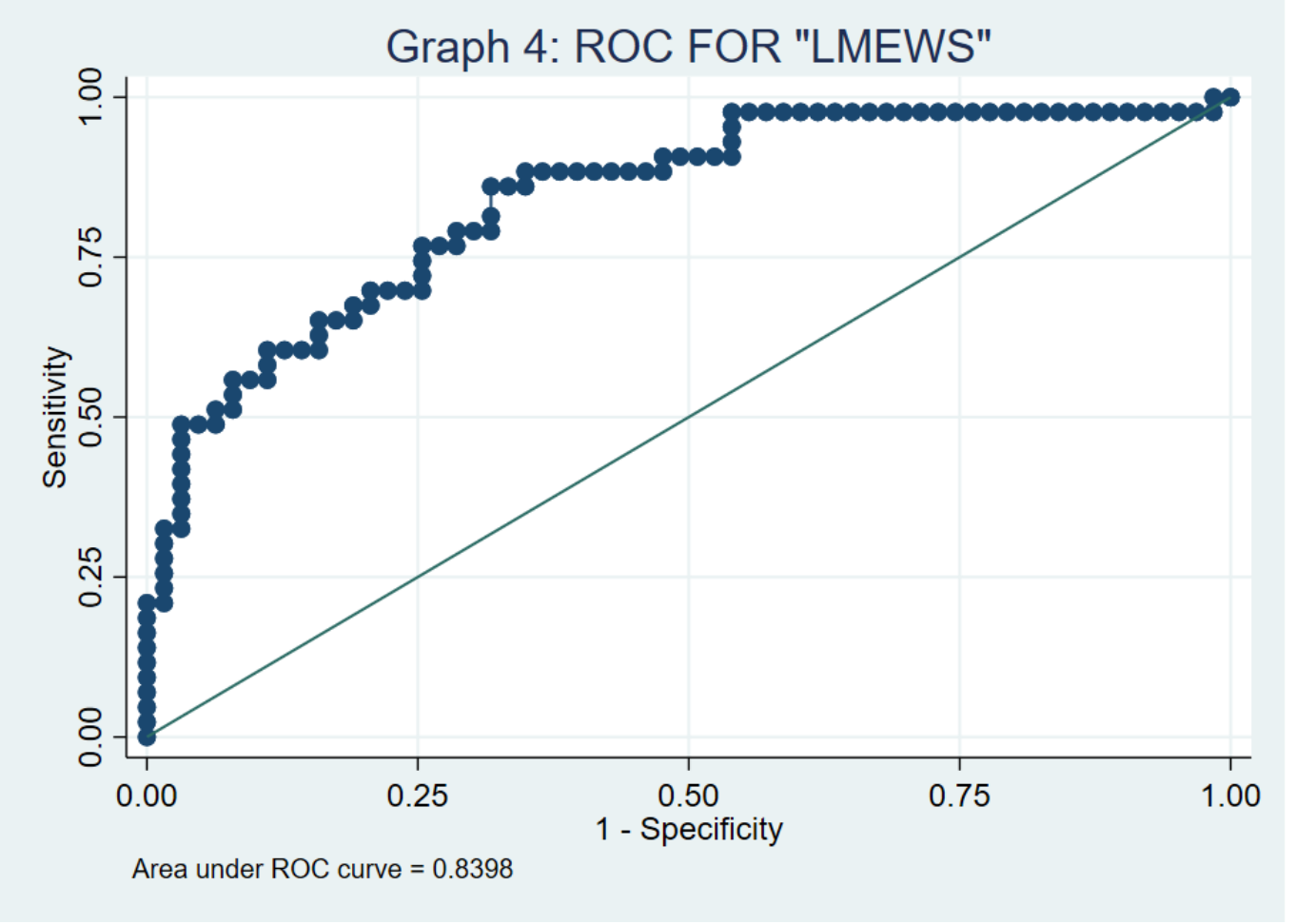

\section{Discussion}

\section{Principal Findings}

MEWS has been validated in several settings as a robust predictor of both clinical deterioration and death in hospital $[2,18]$. This study demonstrates that the approach is useful even in the absence of an observed level of consciousness. Vital signs data collected routinely at the bedside in most facilities in Ghana and throughout sub-Saharan Africa can be used to generate LMEWS, which also has a high predictive value.

Serious adverse events and some portion of in-hospital mortality can be prevented by limiting human error, such as failure to recognize the early warning signs of a deteriorating patient or failure to act on this information in a timely manner [19]. 
MEWS is a low-cost tool that utilizes easy-to-measure bedside parameters to generate a singular value that can identify at-risk patients. This value can be used as a preset trigger in the context of a reporting algorithm.

We found that, in this setting, having a LMEWS value of 4 or greater was highly associated with in-hospital mortality. The area under the curve (AUC) of 0.84 for the LMEWS is consistent with good model accuracy in the discrimination of patients who are critically ill. The combination of LMEWS with clinical judgment is therefore likely to be as effective in Ghana as it has been in other similarly resourced settings [20]. This is encouraging since LMEWS can be implemented without additional training of staff on how to score the level of consciousness and without changing standardized documentation forms already in use for patient monitoring.

The standard inpatient vital signs monitoring charts used in many Ghanaian hospitals includes a 4-hourly graphic to plot temperature, pulse rate, respiratory rate, and blood pressure. Additional parameters may also be serially recorded in some instances or centers; however, the typical bedside observation chart does not record the level of consciousness for patients, as captured in the MEWS by including either the AVPU or RASS score.

Although the original description defined a significant MEWS as any single score $\geq 5$, or any increase of $2+$ points in patients with initial scores above 5, a cut-off of 4 was adopted for this study $[2,16]$. Arguably, a lower threshold for detection would increase the burden of patient re-examination and reassessment on health care providers, potentially making use of the score impractical in settings with severely limited human resources. The decision to adopt a cut-off score of 4 as the definition of a significant MEWS was based on previous work done by Gardner-Thorpe et al [16] in 2006, which showed that raising the threshold reduces the sensitivity to unacceptable levels for patient safety, though an increase in specificity would be observed. Using a cut-off of 4 , the number of individuals with a significant MEWS value was 33 (out of 112), and 31 had a significant LMEWS value. In other words, nearly $30 \%$ of the patients in our study would have been categorized as high risk for clinical deterioration in the context of a MEWS-based reporting algorithm.

Interestingly, using MEWS or LMEWS with a cut-off of $\geq 5$ did not only yield higher discrimination, based on the C-statistics, but also had better calibration in terms of correctly assessing the risk of disease severity. Based on the receiver operating characteristics and the Hosmer-Lemeshow goodness-of-fit test,
LMEWS with a cut-off of $\geq 5$ was superior to both MEWS and LMEWS with a cut-off of $\geq 4$.

Encouraging complete, accurate documentation and a standardized interpretation of vital signs with appropriate actions by nurses, doctors, and other allied staff can potentially improve the outcomes of patients admitted to hospitals, even in a setting that lacks rapid response teams. Many interventions such as fluids or antibiotics do not require advanced equipment or costly supplies, making the implementation of the afferent arm of a rapid response system important even in settings where the efferent arm is more limited [21].

\section{Limitations}

This study is subject to all the limitations of a single-center, retrospective chart review. Sources of bias include the potential for differential clinical care based on perceived patient status in the absence of a standardized rapid response team or protocol. In addition, the study only examined vital signs collected at a single time point for each patient. Changes in serially measured physiological parameters were not evaluated. A study published by Ludikhuize et al [22] recommends the calculation of MEWS at least 3 times daily to detect the development of physiological abnormalities. Our study could not have detected any significant MEWS values that may have developed after the first 48 hours upon admission. However, missing additional patients who may have worsened later and then died would bias the study toward the null hypothesis. This makes our study design a conservative one, with results consistent with previously published literature on the topic $[2,16]$.

More prospective research is needed to help define the utility of LMEWS for physicians looking to allocate resources and develop rapid response teams that can act on predictive information to improve patient outcomes and patient care.

\section{Conclusion}

This study was the first to examine the ability of an early warning system to predict inpatient mortality based on routinely collected clinical data in a low-resource setting. Early recognition of clinical status decline is critical even in low-resource settings, where bedside interventions may prevent ICU admissions and disease complications including death. Though the MEWS system provides good discrimination, the LMEWS provides better discrimination and calibration in the prediction of mortality and can identify critical illness among inpatients with primarily medical diagnoses. Additional prospective studies will be useful to validate LMEWS among other categories of inpatients and to investigate its impact on health resource allocation and clinical outcomes in low-resource settings.

\section{Acknowledgments}

This research was funded by the Leroy Burney Family Fund, which had no role in the design, collection, interpretation of data, or writing and submission of the manuscript.

We are grateful to the Korle-Bu Teaching Hospital, medical department staff, and clientele for making patient charts available for data collection, as well as Drs Benjamin Sena Fenu and Oforiwaa Amoah, who contributed to data collection and on-site follow-up for ethical and technical committee approval; Victor Nortey, who was instrumental in guiding us through the ethical 
review processes at the Korle-Bu Teaching Hospital; and George Mwinnyaa and Seth Bennett, who reviewed the statistical analysis.

\section{Authors' Contributions}

EJA was responsible for the concept, study design, partial data collection, analysis and interpretation, and writing of the manuscript. PA, JSRM, MC, and SES participated in concept development, critical revision, and review of the manuscript. PA, JSRM, and MC also served as academic mentors, while SES was the on-site preceptor as well.

\section{Conflicts of Interest}

None declared.

\section{References}

1. Ghana. The World Bank. URL: https://data.worldbank.org/country/ghana?view=chart [accessed 2019-11-11]

2. Subbe C, Kruger M, Rutherford P, Gemmel L. Validation of a modified Early Warning Score in medical admissions. QJM 2001 Oct;94(10):521-526. [doi: 10.1093/qjmed/94.10.521] [Medline: 11588210]

3. Kruisselbrink R, Kwizera A, Crowther M, Fox-Robichaud A, O'Shea T, Nakibuuka J, et al. Modified Early Warning Score (MEWS) Identifies Critical Illness among Ward Patients in a Resource Restricted Setting in Kampala, Uganda: A Prospective Observational Study. PLoS One 2016 Mar 17;11(3):e0151408 [FREE Full text] [doi: 10.1371/journal.pone.0151408] [Medline: 26986466]

4. Improving Critical Care in Ghana, One Facility at a Time. Knowledge@Wharton Show, University of Pennsylvania. 2017 Mar 8. URL: https://knowledge.wharton.upenn.edu/article/doctor-seeks-to-build-private-acute-care-center-in-ghana/ [accessed 2019-11-08]

5. Schein RM, Hazday N, Pena M, Ruben BH, Sprung CL. Clinical antecedents to in-hospital cardiopulmonary arrest. Chest 1990 Dec;98(6):1388-1392. [doi: 10.1378/chest.98.6.1388] [Medline: 2245680]

6. Kwon J, Lee Y, Lee Y, Lee S, Park J. An Algorithm Based on Deep Learning for Predicting In - Hospital Cardiac Arrest. J Am Heart Assoc 2018 Jul 03;7(13):e008678. [doi: 10.1161/jaha.118.008678] [Medline: 29945914]

7. Kim WY, Shin YJ, Lee JM, Huh JW, Koh Y, Lim C, et al. Modified Early Warning Score Changes Prior to Cardiac Arrest in General Wards. PLoS One 2015 Jun 22;10(6):e0130523 [FREE Full text] [doi: 10.1371/journal.pone.0130523] [Medline: 26098429]

8. Souza BT, Lopes MCBT, Okuno MFP, Batista REA, Góis AFTD, Campanharo CRV. Identification of warning signs for prevention of in-hospital cardiorespiratory arrest. Rev. Latino-Am. Enfermagem 2019;27:e3072. [doi: 10.1590/1518-8345.2853.3072]

9. Ludikhuize J, Smorenburg SM, de Rooij SE, de Jonge E. Identification of deteriorating patients on general wards; measurement of vital parameters and potential effectiveness of the Modified Early Warning Score. J Crit Care 2012 Aug;27(4):424.e7-424.13. [doi: 10.1016/j.jcrc.2012.01.003] [Medline: 22341727]

10. Smith MEB, Chiovaro J, O'Neil M, Kansagora P, Quinones A, Freeman M, et al. Early Warning System Scores: A Systematic Review. In: VA Evidence-based Synthesis Program Reports. Washington DC: Department of Veterans Affairs; $2014: 225$.

11. Andrews T, Waterman H. Packaging: a grounded theory of how to report physiological deterioration effectively. $\mathrm{J}$ Adv Nurs 2005;52(6):443-481. [doi: 10.1111/j.1365-2648.2005.03615.x]

12. Opio MO, Nansubuga G, Kellett J. Validation of the VitalPAC ${ }^{\text {TM }}$ Early Warning Score (ViEWS) in acutely ill medical patients attending a resource-poor hospital in sub-Saharan Africa. Resuscitation 2013 Jun;84(6):743-746. [doi: 10.1016/j.resuscitation.2013.02.007] [Medline: 23438452]

13. Prytherch DR, Smith GB, Schmidt PE, Featherstone PI. ViEWS--Towards a national early warning score for detecting adult inpatient deterioration. Resuscitation 2010 Aug;81(8):932-937. [doi: 10.1016/j.resuscitation.2010.04.014] [Medline: 20637974]

14. Burch VC, Tarr G, Morroni C. Modified early warning score predicts the need for hospital admission and inhospital mortality. Emerg Med J 2008 Oct 01;25(10):674-678. [doi: 10.1136/emj.2007.057661] [Medline: 18843068]

15. Medical Sub-BMC: About Us. Korle Bu Teaching Hospital. URL: http://kbth.gov.gh/departments-centres/ department-of-medicine/ [accessed 2019-11-08]

16. Gardner-Thorpe J, Love N, Wrightson J, Walsh S, Keeling N. The Value of Modified Early Warning Score (MEWS) in Surgical In-Patients: A Prospective Observational Study. Annals 2006 Oct;88(6):571-575. [doi: 10.1308/003588406x130615]

17. Modified Early Warning Score (MEWS), Escalation and ISBAR.. Queensland Government, Queensland Health. URL: https://www.safetyandquality.gov.au/sites/default/files/migrated/ Prince-Charles-Hospital-procedure-MEWS-escalation-and-ISBAR.pdf [accessed 2019-11-08]

18. Kyriacos U, Jelsma J, Jordan S. Record review to explore the adequacy of post-operative vital signs monitoring using a local modified early warning score (mews) chart to evaluate outcomes. PLoS One 2014 Jan 31;9(1):e87320 [FREE Full text] [doi: 10.1371/journal.pone.0087320] [Medline: 24498075] 
19. Wang A, Fang C, Chen S, Tsai S, Kao W. Periarrest Modified Early Warning Score (MEWS) predicts the outcome of in-hospital cardiac arrest. J Formos Med Assoc 2016 Feb;115(2):76-82 [FREE Full text] [doi: 10.1016/j.jfma.2015.10.016] [Medline: 26723861]

20. Wilson RM, Harrison BT, Gibberd RW, Hamilton JD. An analysis of the causes of adverse events from the Quality in Australian Health Care Study. Med J Aust 1999 May 03;170(9):411-415. [doi: 10.5694/j.1326-5377.1999.tb127814.x] [Medline: 10341771]

21. ARISE Investigators and the ANZICS Clinical Trials Group T. Goal-Directed Resuscitation for Patients with Early Septic Shock. N Engl J Med 2014 Oct 16;371(16):1496-1506. [doi: 10.1056/nejmoa1404380]

22. Ludikhuize J, Borgert M, Binnekade J, Subbe C, Dongelmans D, Goossens A. Standardized measurement of the Modified Early Warning Score results in enhanced implementation of a Rapid Response System: a quasi-experimental study. Resuscitation 2014 May;85(5):676-682. [doi: 10.1016/j.resuscitation.2014.02.009] [Medline: 24561029]
Abbreviations
AUC: area under the curve
AVPU: alert, voice, pain, unresponsive
ICU: intensive care unit
IRB: Institutional Review Board
IRR: incidence rate ratio
LMEWS: limited modified early warning score
MEWS: modified early warning score
OR: odds ratio
RASS: Richmond Agitation Sedation Scale
ROC: receiver operating characteristic

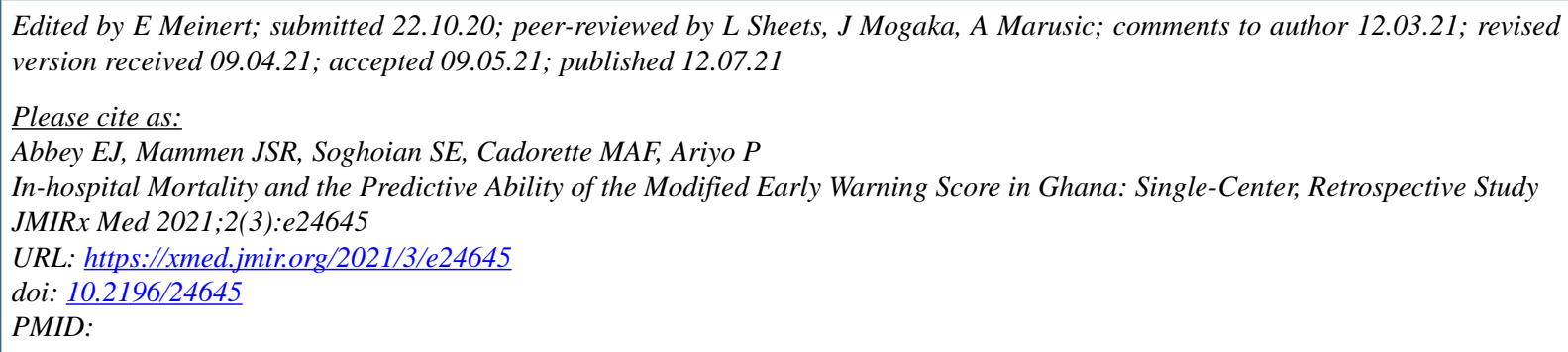

CEnoch Joseph Abbey, Jennifer S R Mammen, Samara E Soghoian, Maureen A F Cadorette, Promise Ariyo. Originally published in JMIRx Med (https://med.jmirx.org), 12.07.2021. This is an open-access article distributed under the terms of the Creative Commons Attribution License (https://creativecommons.org/licenses/by/4.0/), which permits unrestricted use, distribution, and reproduction in any medium, provided the original work, first published in JMIRx Med, is properly cited. The complete bibliographic information, a link to the original publication on https://med.jmirx.org/, as well as this copyright and license information must be included. 